\title{
Comparative activity of the penem antibiotic Sch 34343 against Gram-negative and Gram-positive bacteria with special reference to multiresistant strains
}

\author{
F. H. Kayser, J. Novak and A. Strässle \\ Institute of Medical Microbiology, University of Zürich, 8028 Zürich, Switzerland
}

\begin{abstract}
A new penem antibiotic, Sch 34343 , was shown to be active against a large number of Gram-positive bacteria. The drug inhibited penicillinase-positive and -negative staphylococci equally well, being five times more active than cefamandole and ten times more active than methicallin. Most methicillin-resistant staphylococci were inhibited by concentrations between 0.25 and $4 \mathrm{mg} / \mathrm{l}$, but a small group of highly resistant strains were observed. Sch 34343 was eight times less active than ampicallin and penicillin $G$, but as active as pıperacillın against enterococci. The drug showed excellent activity against various streptococci. Sch 34343 was as bactericidal as flucloxacillın, ampicillin and penicillin $\mathbf{G}$ against staphylococc1, enterococci and streptococci, respectively, in klling kinetic tests Enterobacteriaceae susceptible to third-generation cephalosporins were approximately five times less susceptible to Sch 34343 , but MICs were far below the susceptibility breakpoint. Sch 34343 was equally active against Citrobacter and Enterobacter strains that were highly resistant to third-generation cephalosporins and to aztreonam. Together with thienamycin, this drug seems to be a good alternative for the treatment of infections caused by bacteria resistant to thirdgeneration cephalosporins and to aztreonam.
\end{abstract}

\section{Introduction}

Sch 34343 is a new penem antibiotic which is chemically related to the carbapenem antibiotic thienamycin. This study was undertaken to compare the in-vitro efficacy of Sch 34343 with various other $\beta$-lactam antibiotics. Some 250 clinical isolates of Grampositive organisms were included in the study to provide a good indication of the potential efficacy of the drug in the therapy of Gram-positive infections. Special emphasis was given to the bactericidal activity of the drug against Gram-positive bacteria. The activity of Sch 34343 was also examined against Enterobacteriaceae including a small collection of multiply-resistant organisms, which were resistant to the third-generation cephalosporins, aztreonam and often also to aminoglycosides.

\section{Methods}

\section{Bacteria}

The bacteria used were isolated from clinical material and identufied according to standard procedures. Two hundred and sixty eight Gram-positive organisms were examined. The collection of Gram-negative bacilli comprised Escherichia coli (24), 
Klebsiella pneumoniae (21), K. oxytoca (10), K. ozaenae (4), Enterobacter cloacae (29), Ent. aerogenes (13), Ent. agglomerans (11), Citrobacter freundii (50), Citro. diversus (6), Citrobacter spp. (5), Serratia marcescens (16), Ser. liquefaciens (6), Proteus mirabilis (17), Pr. vulgaris (22), Morganella morganii (4), Providentia rettgeri (7), and Prov. stuartii (6). Strains susceptible to third-generation cephalosporins were fresh clinical isolates. Strains resistant to cephalosporins were derived from worldwide sources.

\section{Antibiotics}

Stock solutions of Sch 34343, imipenem, penicillin G, ampicillin, piperacillin, oxacillin, flucloxacillin, methicillin, cephalothin, cefamandole, cefotaxime, ceftriaxone, cefoperazone, ceftazidime, latamoxef (moxalactam), and aztreonam were prepared in sterile distilled water and either used immediately or stored at $-80^{\circ} \mathrm{C}$, if necessary. All drugs were provided by the pharmaceutical companies.

\section{Minimal inhibitory concentration tests}

Minimal inhibitory concentrations (MICs) were determined by serial two-fold dilutions of antibiotics, usually in Mueller-Hinton agar (BBL) or broth. Pneumococci and haemolytic streptococci were tested on agar containing $1 \%(w / v)$ IsoVitaleX (BBL) and $1 \%(w / v)$ haemoglobin (BBL). The inoculum in agar dilution was about $10^{4}$ per spot, and in macro-broth dilution $5 \times 10^{5}$ to $10^{6}$ per $\mathrm{ml}$. MICs were read after incubation at $35^{\circ} \mathrm{C}$ for $18-24 \mathrm{~h}$. The MIC techniques corresponded to the procedures proposed by the National Committee of Clinical Laboratory Standards (1983).

\section{Bactericidal tests}

The concentrations that kulled $99 \%$ and $99.9 \%$ of the inoculum were determined by plating 100 and $10 \mu \mathrm{l}$ of the broth from each tube of the macro dilution test showing no growth on blood agar. Before plating, $200 \mu \mathrm{l}$ of an enzyme solution containing approximately 8 (if concentrations of drugs were $<10 \mathrm{mg} / \mathrm{l}$ ) or 80 (if concentrations were $>10 \mathrm{mg} / \mathrm{l}$ ) units of penicillin amido- $\beta$-lactamhydrolase E.C. 3.5.2.6. from Bacillus cereus 569/H9 (Genzyme Biochemicals Ltd., Springfield Mill, Maidstone) per $\mathrm{ml}$ were added to the broth tubes and incubated at room temperature for $5 \mathrm{~min}$. This served to destroy any drug and, thus, to avoid carry-over of bacteriostatic activity.

Kinetics of killing was investigated in $20 \mathrm{ml}$ of Mueller-Hinton broth, unsupplemented or supplemented with IsoVitaleX and haemoglobin (see above) when necessary. Four times the MIC of the various drugs were added. At appropriate time intervals, samples were withdrawn, incubated with the $B$. cereus enzyme as described and plated undiluted or in appropriate dilutions.

\section{Population analysis}

Populations of methicillin-resistant staphylococci were analysed by disaggregation of overnight broth cultures (briefly, by ultra sonication at $20 \mathrm{kHz}$ ) and surface inoculation of appropriate dilutions on drug-containing Mueller-Hinton agar plates. Colony counts after $48 \mathrm{~h}$ of incubation at 30 or $37^{\circ} \mathrm{C}$ allowed calculation of the number of viable units among $10^{8} \mathrm{cfu}$ resistant to each concentration of antimicrobial agent. 
Table I. Comparative actuvity of Sch 34343 and other $\beta$-lactam antubiotıcs against Gram-positive bacteria

\begin{tabular}{|c|c|c|c|c|c|c|}
\hline \multirow{2}{*}{$\begin{array}{c}\text { Organusm } \\
\text { (no. of isolates) }\end{array}$} & \multirow[b]{2}{*}{ Drug } & \multicolumn{5}{|c|}{$\operatorname{MIC}(\mathrm{mg} / \mathrm{l})^{\prime}$} \\
\hline & & Mean & Mode & Range & $\mathrm{MIC}_{50}{ }^{b}$ & $\mathrm{MIC}_{90}{ }^{b}$ \\
\hline $\begin{array}{l}\text { Staph. aureus } \\
\text { pencillınase-negative (13) }\end{array}$ & $\begin{array}{l}\text { Sch } 34343 \\
\text { imipenem } \\
\text { penicillin G } \\
\text { methicillin } \\
\text { cefamandole }\end{array}$ & $\begin{array}{l}0.11 \\
0.08 \\
0.02 \\
1 \\
0.21\end{array}$ & $\begin{array}{l}0 \cdot 12 \\
0 \cdot 12 \\
003 \\
1 \\
0 \cdot 25\end{array}$ & $\begin{array}{c}0.06-0.25 \\
0.03-0.12 \\
0.01-0.06 \\
05-2 \\
0.12-0.25\end{array}$ & $\begin{array}{l}0.12 \\
006 \\
001 \\
0.5 \\
012\end{array}$ & $\begin{array}{l}0.12 \\
0.12 \\
0.01 \\
1 \\
0.25\end{array}$ \\
\hline $\begin{array}{l}\text { Staph. aureus } \\
\text { penıcillınase-positıve (20) }\end{array}$ & $\begin{array}{l}\text { Sch } 34343 \\
\text { Imipenem } \\
\text { methicillin } \\
\text { cefamandole } \\
\text { cefotaxime }\end{array}$ & $\begin{array}{l}0 \cdot 15 \\
0 \cdot 09 \\
1.68 \\
0 \cdot 5 \\
22\end{array}$ & $\begin{array}{l}0 \cdot 12 \\
0 \cdot 12 \\
2 \\
05 \\
2\end{array}$ & $\begin{array}{c}0.06-0.5 \\
0.03-0.12 \\
1-2 \\
0.25-2 \\
2-4\end{array}$ & $\begin{array}{l}0.12 \\
0.06 \\
1 \\
1 \\
2\end{array}$ & $\begin{array}{l}0 \cdot 25 \\
0 \cdot 12 \\
2 \\
2 \\
2\end{array}$ \\
\hline $\begin{array}{l}\text { Staph aureus } \\
\text { methicillin-resistant (39) }\end{array}$ & $\begin{array}{l}\text { Sch } 34343 \\
\text { Sch } 34343\left(30^{\circ} \mathrm{C}\right) \\
\text { Imıpenem } \\
\text { methicillın } \\
\text { cefamandole } \\
\text { cefotaxime }\end{array}$ & $\begin{array}{l}0.69 \\
16 \\
0 \cdot 23 \\
4 \cdot 76 \\
19 \\
21 \cdot 2\end{array}$ & $\begin{array}{l}* \\
* \\
* \\
* \\
*\end{array}$ & $\begin{array}{r}012-256 \\
0.5-256 \\
0.06-128 \\
1-256 \\
0 \cdot 25-256 \\
8-256\end{array}$ & $\begin{array}{l}0 \cdot 5 \\
1 \\
012 \\
4 \\
1 \\
32\end{array}$ & $\begin{array}{r}4 \\
4 \\
1 \\
8 \\
4 \\
64\end{array}$ \\
\hline $\begin{array}{l}\text { Staph epidermidis } \\
\text { penıcillınase-negatıve (14) }\end{array}$ & $\begin{array}{l}\text { Sch } 34343 \\
\text { imıpenem } \\
\text { penicillin G } \\
\text { methicillın } \\
\text { cefamandole }\end{array}$ & $\begin{array}{l}0.23 \\
0.07 \\
0.02 \\
1.77 \\
0.27\end{array}$ & $\begin{array}{l}0.12 \\
0.03 \\
0.03 \\
1 \\
0.25\end{array}$ & $\begin{array}{l}0.06-1 \\
0.03-1 \\
0.01-0.12 \\
0.5-8 \\
0.06-1\end{array}$ & $\begin{array}{ll}0 & 25 \\
0 & 06 \\
0 & 01 \\
1 & \\
0 & 12\end{array}$ & $\begin{array}{l}0.5 \\
025 \\
0.06 \\
4 \\
05\end{array}$ \\
\hline $\begin{array}{l}\text { Staph. epidermidis } \\
\text { penıcillınase-positive (27) }\end{array}$ & $\begin{array}{l}\text { Sch } 34343 \\
\text { imipenem } \\
\text { methıcıllin } \\
\text { cefamandole } \\
\text { ceftriaxone }\end{array}$ & $\begin{array}{l}0 \cdot 13 \\
0 \cdot 12 \\
2 \cdot 19 \\
0 \cdot 27 \\
2 \cdot 1\end{array}$ & $\begin{array}{l}0 \cdot 12 \\
0 \cdot 01 \\
2 \\
0 \cdot 25 \\
2\end{array}$ & $\begin{array}{c}0.06-0.25 \\
0.01-0.25 \\
0.5-16 \\
0.06-2 \\
0.25-16\end{array}$ & $\begin{array}{l}012 \\
0.06 \\
1 \\
012 \\
2\end{array}$ & $\begin{array}{l}025 \\
0 \cdot 25 \\
4 \\
05 \\
8\end{array}$ \\
\hline $\begin{array}{l}\text { Siaph. epidermidis } \\
\text { methicillın-resistant (14) }\end{array}$ & $\begin{array}{l}\text { Sch } 34343 \\
\text { imipenem } \\
\text { ceftriaxone }\end{array}$ & $\begin{array}{c}5 \cdot 4 \\
2 \\
176\end{array}$ & * & $\begin{array}{c}1-128 \\
006-32 \\
4-256\end{array}$ & $\begin{array}{l}4 \\
2 \\
8\end{array}$ & $\begin{array}{r}8 \\
16 \\
128\end{array}$ \\
\hline Str faecalis (31) & $\begin{array}{l}\text { Sch } 34343 \\
\text { imipenem } \\
\text { penicillin G } \\
\text { ampicillin } \\
\text { piperacillin } \\
\text { cefamandole }\end{array}$ & $\begin{array}{c}5 \cdot 9 \\
2.4 \\
1 \cdot 9 \\
0.9 \\
5.9 \\
25\end{array}$ & $\begin{array}{r}4 \\
2 \\
2 \\
1 \\
4 \\
32\end{array}$ & $\begin{array}{c}4-32 \\
1-4 \\
1-2 \\
0 \cdot 5-2 \\
1-8 \\
16-32\end{array}$ & $\begin{array}{l}4 \\
2 \\
1 \\
05 \\
4 \\
16\end{array}$ & $\begin{array}{r}8 \\
4 \\
2 \\
1 \\
8 \\
32\end{array}$ \\
\hline
\end{tabular}


Table I. (continued)

\begin{tabular}{|c|c|c|c|c|c|c|}
\hline \multirow{2}{*}{$\begin{array}{c}\text { Organism } \\
\text { (no. of isolates) }\end{array}$} & \multirow[b]{2}{*}{ Drug } & \multicolumn{5}{|c|}{$\operatorname{MIC}(\mathrm{mg} /)^{a}$} \\
\hline & & Mean & Mode & Range & $\mathrm{MIC}_{50}{ }^{\circ}$ & $\mathrm{MIC}_{90}{ }^{b}$ \\
\hline Str. faecium (14) & $\begin{array}{l}\text { Sch } 34343 \\
\text { imipenem } \\
\text { penicillin G } \\
\text { ampicillin } \\
\text { piperacillin } \\
\text { cefamandole }\end{array}$ & $\begin{array}{l}32 \\
6 \cdot 9 \\
3 \cdot 4 \\
0 \cdot 4 \\
13 \cdot 8 \\
74\end{array}$ & * & $\begin{array}{c}4-256 \\
0.03-64 \\
0.25-16 \\
0.5-16 \\
1-64 \\
2-256\end{array}$ & $\begin{array}{r}32 \\
8 \\
2 \\
2 \\
16 \\
64\end{array}$ & $\begin{array}{r}128 \\
32 \\
16 \\
8 \\
32 \\
256\end{array}$ \\
\hline Str. pyogenes (18) & $\begin{array}{l}\text { Sch } 34343 \\
\text { imipenem } \\
\text { penicillin g } \\
\text { cefamandole } \\
\text { ceftriaxone }\end{array}$ & $\begin{array}{l}0.06 \\
0.05 \\
0.01 \\
0.05 \\
0.03\end{array}$ & $\begin{array}{l}0.06 \\
0.03 \\
0.01 \\
0.06 \\
0.03\end{array}$ & $\begin{array}{c}0.03-0.12 \\
0.03-0.12 \\
0.01 \\
0.01-0.12 \\
0.03\end{array}$ & $\begin{array}{l}0.06 \\
0.03 \\
0.01 \\
0.03 \\
0.03\end{array}$ & $\begin{array}{l}0.06 \\
0.12 \\
0.01 \\
0.12 \\
0.03\end{array}$ \\
\hline Str. agalactiae (9) & $\begin{array}{l}\text { Sch } 34343 \\
\text { imipenem } \\
\text { penicillin G } \\
\text { cefamandole } \\
\text { ceftriaxone }\end{array}$ & $\begin{array}{ll}0.15 \\
0.42 \\
0 & 03 \\
0 & 13 \\
0 & 25\end{array}$ & $\begin{array}{l}0 \cdot 12 \\
0 \cdot 5 \\
0 \cdot 03 \\
0 \cdot 12 \\
0.25\end{array}$ & $\begin{array}{c}0.12-0.25 \\
0.25-0.5 \\
0.03 \\
0.03-0.12 \\
0.25\end{array}$ & $\begin{array}{l}0.12 \\
0.5 \\
003 \\
0.06 \\
0.25\end{array}$ & $\begin{array}{l}0.25 \\
0.5 \\
003 \\
0.25 \\
0.25\end{array}$ \\
\hline Str. pneumoniae (28) & $\begin{array}{l}\text { Sch } 34343 \\
\text { imipenem } \\
\text { penicillin G } \\
\text { cefamandole } \\
\text { ceftriaxone }\end{array}$ & $\begin{array}{l}0.07 \\
0.11 \\
0.01 \\
0.03 \\
0.03\end{array}$ & $\begin{array}{l}0.06 \\
0.06 \\
0.01 \\
0.06 \\
0.03\end{array}$ & $\begin{array}{l}0.03-0.5 \\
0.03-0.5 \\
0.01-0.03 \\
0.01-0.25 \\
0.01-0.25\end{array}$ & $\begin{array}{l}0.06 \\
0.06 \\
0.01 \\
0.03 \\
0.03\end{array}$ & $\begin{array}{l}0.25 \\
0.25 \\
0.01 \\
0 \cdot 12 \\
0.03\end{array}$ \\
\hline Corynebacterium JK (12) & $\begin{array}{l}\text { Sch } 34343 \\
\text { imipenem }\end{array}$ & $\begin{array}{l}369 \\
362\end{array}$ & $\begin{array}{l}256 \\
256\end{array}$ & $\begin{array}{l}128-256 \\
128-256\end{array}$ & $\begin{array}{l}256 \\
256\end{array}$ & $\begin{array}{l}256 \\
256\end{array}$ \\
\hline $\begin{array}{l}\text { Corynebacterium } \\
\text { unspecified (34) }\end{array}$ & $\begin{array}{l}\text { Sch } 34343 \\
\text { imipenem }\end{array}$ & $\begin{array}{l}6 \cdot 7 \\
2 \cdot 4\end{array}$ & $*$ & $\begin{array}{l}0.03-256 \\
0.03-256\end{array}$ & $\begin{array}{l}1 \\
0 \cdot 25\end{array}$ & $\begin{array}{l}256 \\
256\end{array}$ \\
\hline
\end{tabular}

- Data obtained with the agar dilution procedure of the National Committee of Clinical Laboratory Standards (1983). Incubation was carried out at $35^{\circ} \mathrm{C}$, unless otherunse indicated, for 18-24 h

- Concentration required to inhibit 50 or $90 \%$ of the examined strauns, respectively

* Wide range of MICs

\section{Results}

Comparison of Sch 34343 with other $\beta$-lactam antibiotics against Gram-positive bacteria

Table I summarizes MICs of Sch 34343 and other drugs against Gram-positive bacteria. As can be seen, the drug showed excellent actıvity against staphylococci, whether they produced penicillinase or not. The drug was 15 times more active than methicillin and exhibited two to four times better activity than cefamandole against methicillin-susceptible cultures. Imipenem was either two to four times more active or showed equal activity to Sch 34343 . Imipenem and Sch 34343 also showed some activity against methicilln-resistant Staph. aureus and Staph. epidermidis, when tested in agar dilution with an inoculum of $10^{4}$ per spot and incubated for $24 \mathrm{~h}$ at $35^{\circ} \mathrm{C}$. Incubation of plates for $48 \mathrm{~h}$ or incubation at $30^{\circ} \mathrm{C}$ increased MICs by a factor of 


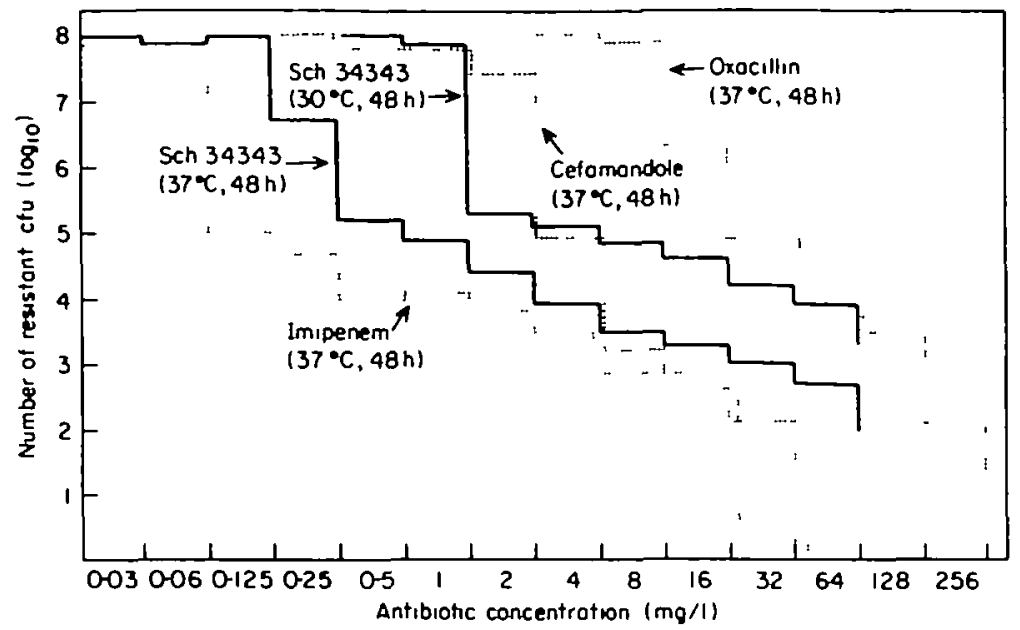

Figure 1. Composition of highly methicilln-resistant Staph aureus strain EK 695 of cells with different levels of resistance to Sch 34343 , imipenem, cefamandole and oxacillın

5-10. These two drugs were slightly more active than cefamandole, which was shown elsewhere to be one of the most active $\beta$-lactam drugs against methicillin-resistant staphylococci (Kayser, 1980).

Heterogeneity amongst the methicillin-resistant staphylococci was observed with Sch 34343 . The drug was more active than cefamandole and oxacillin and of similar activity to imipenem in these population studies (Figure 1).

Although Sch 34343 was not as potent as penicilln or ampicillin against the enterococci, it showed some activity, equal to that of piperacillin, and ten times or more the activity of the cephalosporins. Imipenem also proved to have moderate activity against enterococci.

Sch 34343 and imipenem were equally active against various species of streptococci. However, penicillin $G$ still remained the most potent agent against these organisms.

Although corynebacteria are generally contaminants in microbiological samples, group JK cornynebacterium has been associated with severe infections, particularly in immunosuppressed patients (Young et al., 1981). The organism has also been isolated occasionally from cases of peritonitis in patients undergoing continuous ambulatory peritoneal dialysis (Pierard et al., 1983). These organisms have been found to be resistant to many antimicrobials including the aminoglycosides and $\beta$-lactam antibiotics. JK cornynebacteria were also found to be highly resistant to Sch 34343 and to imipenem. Other unspecified corynebacteria, which were 1solated from infectious lesions and, thus, might have played a role in disease, exhibited straindependent susceptibility or resistance to both drugs.

\section{Bactericidal activity of Sch 34343}

Examination of the bactericidal activity of Sch 34343 and comparative agents was carried out in conventional MBC tests (Anhalt, Sabath \& Barry, 1980) in triplicate Although great care was taken in performing these tests, results were often inconsistent, due to multiple methodological problems (Taylor et al., 1983; Kayser \& 
Table II. Bactericidal activity of Sch 34343 in companson with penicillin G, ampicillin and flucloxacillin against Gram-positive bacteria

\begin{tabular}{|c|c|c|c|c|c|c|}
\hline \multirow[b]{2}{*}{ Organism } & \multicolumn{2}{|c|}{$\operatorname{MIC}(\mathrm{mg} / 1)^{a}$} & \multicolumn{2}{|c|}{$\mathrm{MBC}_{99}\left(\mathrm{mg} / \mathrm{l}^{b}\right.$} & \multicolumn{2}{|c|}{$\mathrm{MBC}_{99 \cdot 9}(\mathrm{mg} / \mathrm{l})^{b}$} \\
\hline & $\begin{array}{c}\text { Sch } \\
34343\end{array}$ & $\begin{array}{l}\text { Comparative } \\
\text { drug }\end{array}$ & $\begin{array}{c}\text { Sch } \\
34343\end{array}$ & $\begin{array}{c}\text { Comparative } \\
\text { drug }\end{array}$ & $\begin{array}{c}\text { Sch } \\
34343\end{array}$ & $\begin{array}{c}\text { Comparatıve } \\
\text { drug }\end{array}$ \\
\hline \multirow{4}{*}{$\begin{array}{l}\text { Staph. aureus } 41 \\
\text { penicillinase-positive } \\
\text { Staph. aureus } 61 \\
\text { penicillinase-negatuve } \\
\text { Staph. epidermidis } 145 \\
\text { penicillinase-positive } \\
\text { Staph. epidermidis } 162 \\
\text { penicillinase-negative }\end{array}$} & $0 \cdot 12$ & 0.25 & 2 & 0.25 & $>64$ & $>64$ \\
\hline & 0.06 & 0.25 & 025 & 025 & $>64$ & 0.25 \\
\hline & 0.06 & 0.25 & 1 & 1 & 32 & 4 \\
\hline & $0 \cdot 12$ & 0.5 & 1 & 1 & 1 & 16 \\
\hline $\begin{array}{l}\text { Str. faecalis } 97 \\
\text { Str. faecium } 128\end{array}$ & $\begin{array}{r}8 \\
16\end{array}$ & $\begin{array}{l}1 \\
2\end{array}$ & $\begin{array}{r}64 \\
>64\end{array}$ & $\begin{array}{r}32 \\
>64\end{array}$ & $\begin{array}{l}>64 \\
>64\end{array}$ & $\begin{array}{l}>64 \\
>64\end{array}$ \\
\hline $\begin{array}{l}\text { Str. pyogenes } 193 \\
\text { Str agalactiae } 219 \\
\text { Str. pneumoniae } 241 \\
\text { Str. mutans } 349\end{array}$ & $\begin{array}{l}0.12 \\
0.12 \\
0.01 \\
0.06\end{array}$ & $\begin{array}{l}0.03 \\
0.06 \\
0.03 \\
0.03\end{array}$ & $\begin{array}{l}1 \\
1 \\
0.03 \\
0.25\end{array}$ & $\begin{aligned} & 0.03 \\
& 0.25 \\
& 0.01 \\
&>64\end{aligned}$ & $\begin{array}{c}>64 \\
1 \\
003 \\
>64\end{array}$ & $\begin{aligned} & 0.25 \\
& 0.5 \\
& 001 \\
> & 64\end{aligned}$ \\
\hline
\end{tabular}

\footnotetext{
- Minimal inhibitory concentration determined in macro-broth dilution according to the NCCLS procedure.

- Concentration necessary to kill 99 or $999 \%$ of the inoculum, respectively.

- Comparatuve drug for staphylococa was fluctoxacillin, for enterococci ampicillin and for streptococes penicallin G.
}

Müller, 1983). Table II summarizes some of the results obtained. With the exception of enterococci, Sch 34343 showed good bactericidal activity at the $99 \%$ killing level. At the $99.9 \%$ level, however, it was often not bactericidal even at high concentrations. Sometimes the comparative agent also was not bactericidal at this level. Sometimes Sch 34343 was active, but the comparative drug was not. No paradoxical effect (Eagle \& Musselman, 1948) was observed with strains and drugs examined. We therefore decided to study the bactericidal potential of Sch 34343 in a kinetic system. Figure 2 shows that Sch 34343 extensively killed the organisms examined with a rate identical to that of the comparative drugs.

\section{Comparison of Sch 34343 with other $\beta$-lactam antibiotics against Gram-negative bacteria}

Recent reports have shown that Enterobacteriaceae can become resistant to thirdgeneration cephalosporins by mutation in a chromosomal locus, regulating the production of a cephalosporinase (Seeberg, Tolxdorff-Neutzling \& Wiedemann, 1983). The frequency of resistant mutants of this type in clinical isolates is still low (Kayser \& Kohler, 1984). They have mainly been observed in Citrobacter and Enterobacter species. We collected such strains from our area and from worldwide sources and examined the activity of Sch 34343 against them. Table III summarizes the results obtained. For comparative purposes, data about the activity of the advanced $\beta$-lactam antibiotics against strains of Enterobacteriaceae susceptible to third-generation 


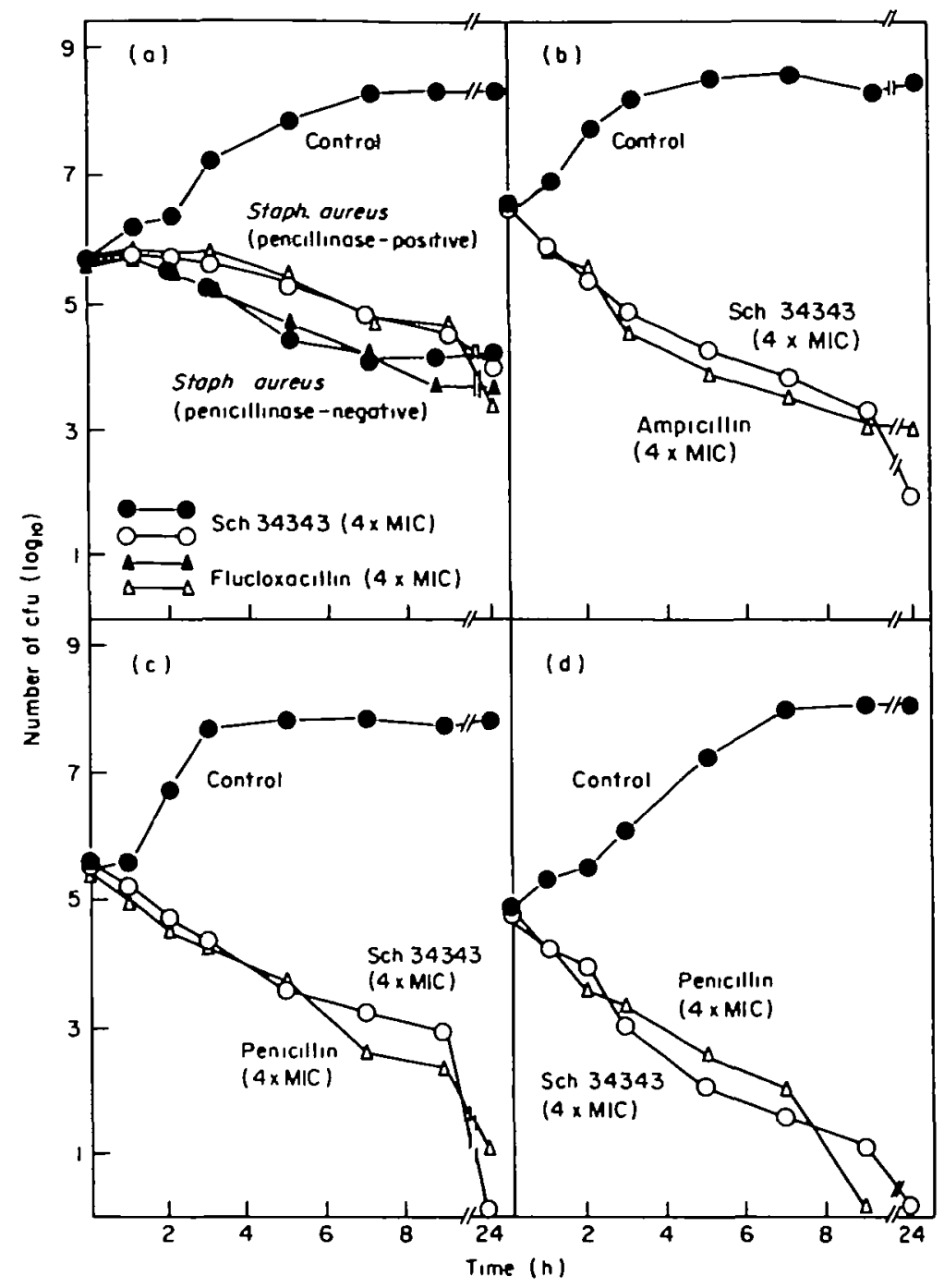

Figure 2. Rates of kulhng of Staph. aureus, Str. faecals, Sir. pyogenes and Str. preumonice by Sch 34343 $(\mathrm{O}-\mathrm{O})$ and fuclocacillin $(\Delta-\Delta)$, ampicilın $(\Delta-\Delta)$ and pencallin $\mathrm{G}(\Delta-\Delta)$, respectuvely (a) Open symbols: Siaph aureus 41 (peniallınase-posituve) MIC of Sch $34343=0125$ and of flucloxacillin $=0.25 \mathrm{mg} /$ Closed symbols Staph. aureus 61 (penicillınase negative). MIC of Sch $34343=006$ and flucloxacillon $=025 \mathrm{mg} / \mathrm{l}$ (b) Str faecalss 97 MIC of Sch $34343=8$ and of ampicilın $=1 \mathrm{mg} / \mathrm{l}$. (c) Str. pyogenes $193 \mathrm{MIC}$ of Sch $34343=0.125$ and of penicullın $\mathrm{G}=0.03 \mathrm{mg} /$ (d) Sir pneumontae 241. MIC of Sch $34343=0.008$ and of penicillı $\mathrm{G}=0.03 \mathrm{mg} / \mathrm{l}$

cephalosporins have been included. It can be seen that Sch 34343 was not as active as third-generation cephalosporins and aztrenonam on a weight basis against susceptible organisms. MICs of Sch 34343, however, were in a range easily obtained in tissue by normal dosing (Investigational Brochure, Schering Corp., Kenilworth, NJ). However, Sch 34343 and imipenem were only slightly less active against organisms highly resistant to third-generation cephalosporins and to aztreonam, than against the susceptible group. 
Table III. Companson of Sch 34343 with other $\beta$-lactam antibiotics against Gram-negative bacteria susceptible or resistant to third-generation cephalosponns

\begin{tabular}{|c|c|c|c|c|c|c|c|}
\hline \multirow{2}{*}{$\begin{array}{c}\text { Organism } \\
\text { (no. of isolates) }\end{array}$} & \multirow[b]{2}{*}{ Drug } & \multicolumn{3}{|c|}{$\begin{array}{l}\text { MICs }(\mathrm{mg} / \mathrm{l}) \text { of } \\
\text { susceptible isolates }\end{array}$} & \multicolumn{3}{|c|}{$\begin{array}{l}\text { MICs }(\mathrm{mg} / \mathrm{l}) \text { of } \\
\text { resistant isolates }\end{array}$} \\
\hline & & Mean & Mode & Range & Mean & Mode & Range \\
\hline $\begin{array}{l}E . \text { coli } \\
\text { no. sensitive (24) }\end{array}$ & $\begin{array}{l}\text { Sch } 34343 \\
\text { imipenem } \\
\text { cefotaxime } \\
\text { cefoperazone } \\
\text { ceftriaxone } \\
\text { ceftazidime } \\
\text { latamoxef } \\
\text { aztreonam }\end{array}$ & $\begin{array}{l}0.6 \\
0.2 \\
0.1 \\
1.7 \\
01 \\
0.3 \\
0.2 \\
0.2\end{array}$ & $\begin{array}{l}1 \\
0.12 \\
0.12 \\
* \\
0.12 \\
0.12 \\
0.25 \\
0.12\end{array}$ & $\begin{array}{l}0 \cdot 12-16 \\
006-1 \\
003-2 \\
0 \cdot 12-64 \\
0 \cdot 03-0 \cdot 25 \\
0.03-2 \\
0 \cdot 06-0 \cdot 25 \\
0 \cdot 03-1\end{array}$ & & & \\
\hline $\begin{array}{l}\text { Klebsiella spp. } \\
\text { no. sensituve (34) } \\
\text { no. resistant (1) }\end{array}$ & $\begin{array}{l}\text { Sch } 34343 \\
\text { imipenem } \\
\text { cefotaxime } \\
\text { cefoperazone } \\
\text { ceftriaxone } \\
\text { ceftazidime } \\
\text { latamoxef } \\
\text { aztreonam }\end{array}$ & $\begin{array}{l}0 \cdot 3 \\
0 \cdot 2 \\
0 \cdot 1 \\
4 \cdot 7 \\
0.3 \\
0.4 \\
0.3 \\
06\end{array}$ & $\begin{array}{l}0.25 \\
0.25 \\
0.06 \\
* \\
0.06 \\
0.25 \\
0.06 \\
0.06\end{array}$ & $\begin{array}{l}0 \cdot 12-1 \\
0 \cdot 12-1 \\
001-2 \\
0 \cdot 12-256 \\
0.03-64 \\
0.06-2 \\
0.03-2 \\
0.03-64\end{array}$ & $\begin{array}{l}1 \\
0 \cdot 5 \\
16 \\
16 \\
16 \\
16 \\
16 \\
4\end{array}$ & & \\
\hline $\begin{array}{c}\text { Enterobacter spp. } \\
\text { no. sensitive (27) } \\
\text { no. resistant (26) }\end{array}$ & $\begin{array}{l}\text { Sch } 34343 \\
\text { imipenem } \\
\text { cefotaxime } \\
\text { cefoperazone } \\
\text { ceftriaxone } \\
\text { ceftazdime } \\
\text { latamoxef } \\
\text { aztreonam }\end{array}$ & $\begin{array}{l}0.7 \\
0.4 \\
0 \cdot 2 \\
0.3 \\
0.2 \\
0.6 \\
0.3 \\
0.2\end{array}$ & $\begin{array}{l}0.5 \\
0.25 \\
* \\
0.25 \\
0.12 \\
0.25 \\
0.12 \\
0.12\end{array}$ & $\begin{array}{l}0.12-4 \\
0.06-4 \\
0.01-8 \\
0.01-16 \\
0.01-16 \\
0.06-64 \\
0.06-32 \\
0.03-16\end{array}$ & $\begin{array}{r}2 \cdot 1 \\
0 \cdot 4 \\
85 \cdot 6 \\
36 \cdot 5 \\
73 \cdot 0 \\
50 \cdot 2 \\
9.9 \\
26 \cdot 5\end{array}$ & $\begin{array}{c}1 \\
0 \cdot 5 \\
128 \\
32 \\
128 \\
64 \\
8 \\
*\end{array}$ & $\begin{array}{c}0 \cdot 5-16 \\
0 \cdot 12-2 \\
16->256 \\
2->256 \\
8->256 \\
4->256 \\
1-128 \\
1-256\end{array}$ \\
\hline $\begin{array}{l}\text { Serratıa spp. } \\
\text { no. sensituve }(21) \\
\text { no resistant }(1)\end{array}$ & $\begin{array}{l}\text { Sch } 34343 \\
\text { Imipenem } \\
\text { cefotaxime } \\
\text { cefoperazone } \\
\text { ceftriaxone } \\
\text { ceftazidıme } \\
\text { latamoxef } \\
\text { aztreonam }\end{array}$ & $\begin{array}{l}1.8 \\
0.4 \\
0.4 \\
8 \cdot 8 \\
0.3 \\
0.3 \\
0.6 \\
0.3\end{array}$ & $\begin{array}{l}2 \\
0.25 \\
0.25 \\
16 \\
0.12 \\
0.06 \\
0.25 \\
0.12\end{array}$ & $\begin{array}{l}0.5-16 \\
0.25-4 \\
0.06-8 \\
0.5-64 \\
0.03-8 \\
0.06-16 \\
0.06-256 \\
0.06-4\end{array}$ & $\begin{array}{r}16 \\
1 \\
16 \\
16 \\
4 \\
16 \\
256 \\
2\end{array}$ & & \\
\hline $\begin{array}{l}\text { Citrobacter spp. } \\
\text { no. sensitive (43) } \\
\text { no. resistant (18) }\end{array}$ & $\begin{array}{l}\text { Sch } 34343 \\
\text { imipenem } \\
\text { cefotaxime } \\
\text { cefoperazone } \\
\text { ceftriaxone } \\
\text { ceftazidime } \\
\text { latamoxef } \\
\text { aztreonam }\end{array}$ & $\begin{array}{l}0 \cdot 5 \\
0 \cdot 3 \\
0 \cdot 2 \\
1 \cdot 1 \\
0 \cdot 1 \\
0 \cdot 45 \\
0 \cdot 2 \\
0 \cdot 2\end{array}$ & $\begin{array}{l}0.5 \\
0.25 \\
0.12 \\
0.25 \\
0.06 \\
0.25 \\
0.12 \\
0.06\end{array}$ & $\begin{array}{l}0.12-2 \\
012-1 \\
0.01-8 \\
0.06-32 \\
0.01-16 \\
0.06-16 \\
0.06-8 \\
0.03-32\end{array}$ & $\begin{array}{r}0 \cdot 8 \\
0 \cdot 3 \\
38 \cdot 5 \\
47 \cdot 2 \\
40 \cdot 2 \\
38 \cdot 8 \\
3 \cdot 7 \\
13 \cdot 2\end{array}$ & $\begin{array}{l}1 \\
0 \cdot 25 \\
32 \\
32 \\
32 \\
* \\
*\end{array}$ & $\begin{array}{l}0 \cdot 25-4 \\
0 \cdot 12-1 \\
16-128 \\
16->256 \\
16-256 \\
0.06->256 \\
012-64 \\
0 \cdot 25->256\end{array}$ \\
\hline
\end{tabular}


Table III. (continued)

\begin{tabular}{|c|c|c|c|c|c|c|c|}
\hline \multirow{2}{*}{$\begin{array}{c}\text { Organism } \\
\text { (no. of isolates) }\end{array}$} & \multirow[b]{2}{*}{ Drug } & \multicolumn{3}{|c|}{$\begin{array}{l}\text { MICs (mg/l) of } \\
\text { susceptible isolates }\end{array}$} & \multicolumn{3}{|c|}{$\begin{array}{l}\text { MICs }(\mathrm{mg} / \mathrm{l}) \text { of } \\
\text { resistant isolates }\end{array}$} \\
\hline & & Mean & Mode & Range & Mean & Mode & Range \\
\hline $\begin{array}{l}\text { Pr. mirabulis } \\
\quad \text { no. sensitive (17) }\end{array}$ & $\begin{array}{l}\text { Sch } 34343 \\
\text { imipenem } \\
\text { cefotaxime } \\
\text { cefoperazone } \\
\text { ceftriaxone } \\
\text { ceftazidıme } \\
\text { latamoxef } \\
\text { aztreonam }\end{array}$ & $\begin{array}{l}0.9 \\
0.7 \\
0.02 \\
0.4 \\
0.01 \\
0.04 \\
0.1 \\
0.02\end{array}$ & $\begin{array}{l}0.5 \\
0.5 \\
0.01 \\
0.25 \\
0.01 \\
0.03 \\
0.12 \\
0.01\end{array}$ & $\begin{array}{l}0.25-2 \\
0.06-4 \\
0.01-0.06 \\
0.12-8 \\
0.01 \\
0.01-0.5 \\
0.06-0.25 \\
0.01-0.03\end{array}$ & & & \\
\hline $\begin{array}{l}\text { Pr. vulgarls } \\
\text { Morg. morganii } \\
\text { Providencia spp. } \\
\text { no. sensitive (39) }\end{array}$ & $\begin{array}{l}\text { Sch } 34343 \\
\text { imipenem } \\
\text { cefotaxime } \\
\text { cefoperazone } \\
\text { ceftriaxone } \\
\text { ceftazidıme } \\
\text { latamoxef } \\
\text { aztreonam }\end{array}$ & $\begin{array}{l}0.8 \\
0.6 \\
0.02 \\
0.6 \\
0.02 \\
0.05 \\
0.4 \\
0.04\end{array}$ & $\begin{array}{l}0.5 \\
1 \\
0.01 \\
0.5 \\
0.01 \\
0.03 \\
0.25 \\
0.01\end{array}$ & $\begin{array}{l}0.5-4 \\
0.03-4 \\
0.01-0.25 \\
0.06-8 \\
0.01-0.25 \\
0.01-2 \\
0.03-0.5 \\
0.01-4\end{array}$ & & & \\
\hline
\end{tabular}

- Number of isolates susceptible (MIC $\leqslant 8 \mathrm{mg} /$ ) or ressistant (MIC $>8 \mathrm{mg} /$ ) to cefotaxime.

* Wide range of MICs.

\section{Discussion}

Sch 34343 has been shown in this study to be an excellent antibiotic against most species of Gram-positive bacteria. In particular, it exhibited some activity against methicillin-resistant staphylocooci, although the typical characteristics of these bacteria-heterogeneity and influence of incubation temperature on phenotypic expression of resistance-were also observed with Sch 34343. The drug was only three to four times less active than imipenem against methicillin-resistant staphylococci in agar dilution tests, but the numbers of sub-populations resistant to each drug were the same (see Figure 1). These two drugs, therefore, could be considered as a possible choice in the treatment of infections caused by such bacteria in exceptional cases, if for instance other antimicrobials such as vancomycin, could not be used. The general rule, however, that infections by methicillin-resistant staphylocooci should not be treated with any $\beta$-lactam agent, is valid for Sch 34343 and imipenem. Sch 34343 exhibited high activity against streptococci and pneumococci, but enterococci were not as susceptible as towards penicillin G or ampicillin. Against Streptococcus faecalis, however, the drug was as active as piperacillin.

Sch 34343 was as bactericidal as comparative agents (flucloxacillin, ampicillin and penicillin (G) against representative Gram-positive bacteria in the kinetic studies. The variable results obtained with $S c h 34343$ and the comparative drugs in conventional MBC $99.9 \%$ tests reflect the laboratory problems connected with this procedure (Taylor et al., 1983; Kayser \& Müller, 1983).

Although Sch 34343 was approximately five times less active than third-generation cephalosporins against Enterobacteriaceae, MICs were far below the susceptibility 
threshold. MICs were not (or only by one dilution) elevated against Citrobacter and Enterobacter strains, highly resistant to third-generation cephalosporins. This behaviour reflects the extreme stability of the drug to $\beta$-lactamases, since it has been shown that cephalosporin resistance in such strains is due to chromosomal cephalosporinase (Sanders \& Sanders 1983, Seeberg et al., 1983). Sch 34343, thus, together with imipenem, can be considered as potent chemotherapeutic agents in infections caused by Enterobacteriaceae resistant to third-generation cephalosporins and to aztreonam.

\section{References}

Anhalt, J. P., Sabath, S. D \& Barry, A. L. (1980). Special tests: bactericidal activity, activity of antimicrobics in combination and detection of $\beta$-lactamase production In Manual of Clinical Microbiology (E. H. Lennette, A. Balows, W. J. Hausler Jr. \& J. P. Truant, Eds), pp. 478-84. American Society of Microbiology, Washington D.C.

Eagle, H. \& Musselman. A. D. (1948). The rate of bactericidal action of penicillin in vitro as a function of its concentration, and its paradoxically reduced activity at high concentrations against certain organisms. Journal of Experimental Medicine 88, 99-131.

Kayser, F. H. (1980). Die Resistenz Methicillın-resistenter Staphylokokken gegenüber neuen Cephalosporin-Antibiotika Infection 8, 165-70.

Kayser, F. H. \& Müller, F. (1983). Resistance and tolerance of the staphylococcus to $\beta$-lactam antibiotics. In Proc. 13th International Congress of Chemotherapy, Vienna, 1983 (K. H. Spitzy \& K. Karrer, Eds), Symposium no. 45), Staphylococcal Infections, pp. 8-14.

Kayser, F. H. \& Kohler, M. (1984). Vergleich der antibaktenellen Aktivitäten der Cephalosporine. Schwelzerische Medizinische Wochenschrift 114, 156-61.

National Committee of Clinical Laboratory Standards (NCCLS) (1983). Methods for dilution antimicrobial susceptibility tests for bacteria that grow aerobically. Tentative Standard M7T. The National Committee of Clinical Laboratory Standards, Vol. 3, No. 2.

Pierard, D., Lauwers, S., Mouton, M., Sennesael, J. \& Verbeelen, D. (1983). Group JK corynebacterium peritonitis in a patient undergoing contınuous ambulatory peritoneal dialysis. Journal of Clinical Microbiology 18, $1011-4$.

Sanders, C. C. \& Sanders, W. E (1983). Emergence of resistance during therapy with the newer $\beta$-lactam antibiotics: role of inducible $\beta$-lactamases and implications for the future. Review of Infectious Diseases 5, 639-48.

Seeberg, A. H., Tolxdorff-Neutzling, R. M. \& Wiedemann, B. (1983). Chromosomal $\beta$-lactamase of Enterobacter cloacae are responsible for resistance to third-generation cephalosponns. Antumicrobial Agents and Chemotherapy 23, 918-25

Taylor, P. C., Schoenknecht, F. D., Sherris, J. C. \& Linner, E. C. (1983). Determination of minimum bactericidal concentration of oxacillin for Staphylococcus aureus: influence and significance of technical factors. Antimicrobial Agents and Chemotherapy 23, 142-50.

Young, V. M., Meyers, W. F., Moody, M.R. \& Schimpff S. C. (1981). The emergence of coryneform bactena as a cause of nosocomial infection in compromised hosts. American Journal of Medicine 70, 646-50. 